EESTI NSV TEADUSTE AKADEEMIA TOIMETISED.

FOOSIKA * MATEMAATIKA

ИЗВЕСТИЯ АКАДЕМИИ НАУК ЭСТОНСКОП ССР. ФИЗИКА * МАТЕМАТИКА

PROCEEDINGS OF THE ACADEMY OF SCIENCES OF THE ESTONIAN SSR.

PHYSICS * MATHEMATICS

$1985,34,1$

удК 530.145 .6

Т. ПЕИПМАН, Ю. ЭНГЕЛЬБРЕХТ

\title{
К ВОПРОСУ ФОРМИРОВАНИЯ СОЛИТОНОВ
}

\author{
(Представил Н. Алумяэ)
}

Аналитическое описание солитонов является, несомненно, одним из выдающихся достижений математической физики в последние десятилетия [ ${ }^{1}$ ]. Математический аппарат, развитый в связи с необходимостью решения нелинейных задач, и богатая физическая сущность нелинейных процессов позволяют применить концепцию уединенных волн в весьма различных областях физики - в квантовой механике, в физике плазмы, в гидродинамике и т. д. $\left[{ }^{2,3}\right]$. Чаще всего солитоны можно истолковать как решения знаменитого одномерного уравнения Кортевега-де Вриза (КдВ)

$$
\frac{\partial u}{\partial \tau}+u \frac{\partial u}{\partial \zeta}+\mu \frac{\partial^{3} u}{\partial \zeta^{3}}=0,
$$

записанного в системе сопутствующих координат $\tau, \zeta\left[{ }^{4}\right]$. Здесь $u$ можно рассматривать как скорость и $\mu$ - как параметр дисперсии. Автомодельные решения уравнения (1) типа уединенных волн

$$
u=u_{\infty}+a \operatorname{sech}^{2}\left\{\Delta\left[\zeta-\left(u_{\infty}+1 / 3 a\right) \tau\right]\right\}
$$

найдены уже раньше $\left[{ }^{5}\right]$ и в настоящее время свойства их хорошо изучены $\left[{ }^{2,3}\right]$. Здесь $u_{\infty}-$ значение амплитуды в бесконечности («нулевая линия» солитона), $a-$ амплитуда солитона, $\Delta^{2}=1 / 12 a \mu^{-1}$.

В физических процессах, однако, начальные условия (воздействия) не всегда соответствуют автомодельным решениям, поэтому формирование солитонов в ходе распространения волны приобретает важное значение. Первый пример решения уравнения (1) с неавтомодельным начальным условием приведен Н. Дж. Забуским $\left[{ }^{6}\right]$ в случае синусоидального (монохроматического) воздействия. Им показано образование совокупности солитонов при $\mu^{1 / 2}=0,022$ и изучено дальнейшее взаимодействие их в процессе распространения. Далее, Ю. А. Березиным $\left[{ }^{7,8}\right]$ решена задача об эволюции воздействий типа

$$
\begin{aligned}
& u(\zeta, 0)=u_{0} \exp \left(-\zeta^{2} l^{-2}\right), \\
& u(\zeta, 0)=u_{0} \zeta \exp \left(-\zeta^{2} l^{-2}\right),
\end{aligned}
$$

где $u_{0}$ - максимальная амплитуда, $l$ - линейный размер.

Обозначая главный момент воздействия через

$$
S_{1}=\int_{-\infty}^{\infty} u(\zeta, 0) d \zeta
$$

легко видеть, что в случае воздействия (3) имеем $S_{1} \neq 0$, в случае же воздействия (4) $-S_{1}=0$. Число солитонов, образующихся из импульсообразного воздействия (3), зависит от значения $\mu$. При меньших $\mu$ воздействие распадается на большее число солитонов $\left[{ }^{7}\right]$. Такие же ре- 


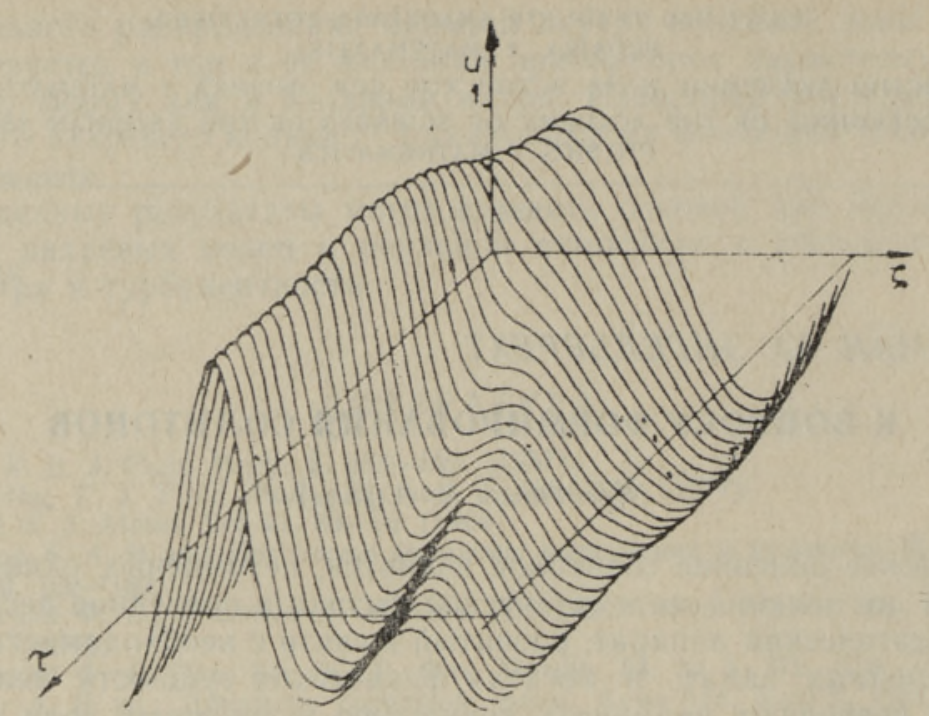

Рис. 1. Эволюция профиля солитонов при $\mu=-0,15$.

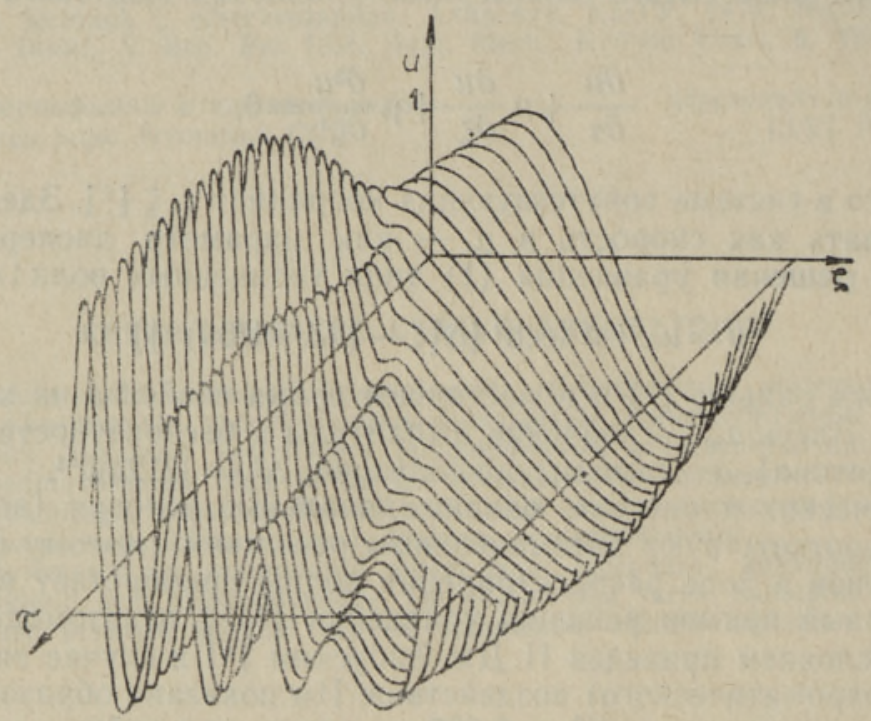

Рнс. 2. Эволюция профиля солитонов прп $\mu=-0,0222$.

зультаты получены в предельном случае решения уравнения КдВ Бюргерса для воздействия (3) при стремлении коэффициента вязкости к нулю $\left[{ }^{9}\right]$. Весь указанный анализ базируется на численном эксперименте (метод конечных разностей), в результате чего определены значения параметра $\mu$, при которых формируется определенное число солитонов или осцилляторный цуг. Аналитическое описание эволюции произвольного начального воздействия до формирования солитонов, по нашим данным, пока отсутствует. Это вполне понятно, потому что согласно методу обратной задачи рассеяния $\left[{ }^{3}\right]$ для получения замкнутого решения необходимо на промежуточном этапе решить интегральное уравнение Гельфанда-Левитана-Марченко, что представляет определенные трудности математического характера. Необходимо, однако, отметить, 


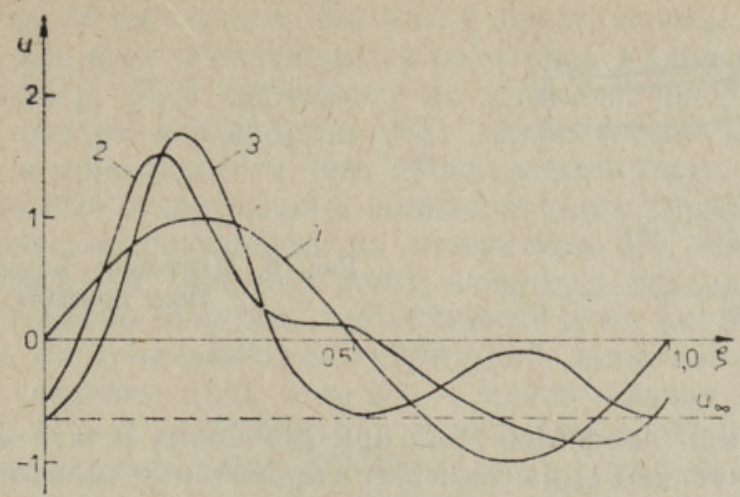

Рис. 3. Профили (при $\mu=-0,15$ ) в различные моменты: $I-\tau=0$ (начальное условие), $2-\tau=1,0,3-\tau=3,0$.

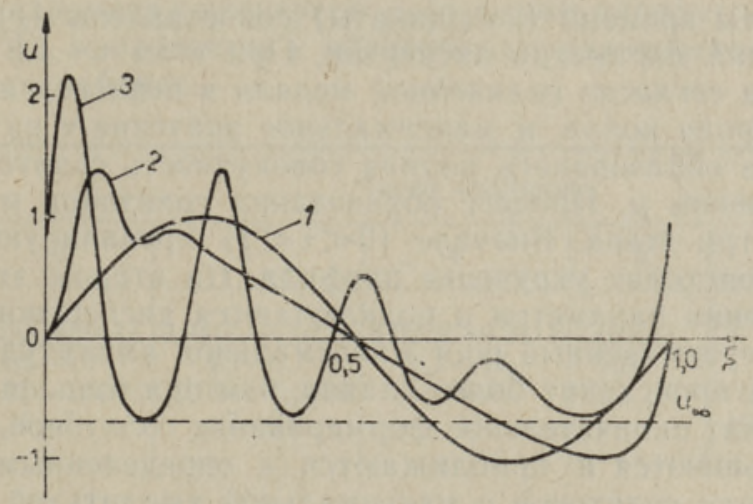

Рнс. 4. Профили (при $\mu=-0,0222$ ) в различные моменты: $I-\tau=0$ (начальное условие), $2-\tau=1,05,3-\tau=2,625$.

что при помощи законов сохранения уравнения КдВ можно определить амплитуды солитонов при известном числе образованных солитонов $\left[{ }^{7}\right]$.

Если в случае воздействий типа (3) и (4) механизм формирования солитонов вполне понятен $\left[{ }^{7,8}\right]$, то при монохроматическом импульсе известен лишь классический результат Забуского для определенного значения $\mu\left[{ }^{6}\right]$, который воспроизводится из одного обзора в другой. Далее детально рассмотрим вопрос формирования солитонов именно в случае монохроматического воздействия. Уравнение (1) с начальным условием

$$
u(\zeta, 0)=\sin 2 \pi \zeta
$$

решается численно псевдоспектральным методом. Алгоритм указанного метода базируется на сочетании методов быстрого преобразования Фурье и конечных разностей $\left[{ }^{10,11}\right]$. Отметим, что уравнение (1) безразмерное и параметр дисперсии $\mu$ имеет структуру

$$
\mu=l_{0}^{2} c_{0}\left(k \tau_{c}^{2} u_{0}\right)^{-1},
$$

где $l_{0}-$ масштабный параметр, $c_{0}-$ скорость звука, $k-$ нелинейный параметр, $\tau_{c}-$ длина волны, $u_{0}-$ максимальная амплитуда $\left[{ }^{4}\right]$.

Образов ан и е соли тонов. Число солитонов $n$, образующихся при начальном монохроматическом воздействии, в одном периоде зависит от значения параметра дисперсии $\mu$, как и в случае воздействий (3) и (4). 


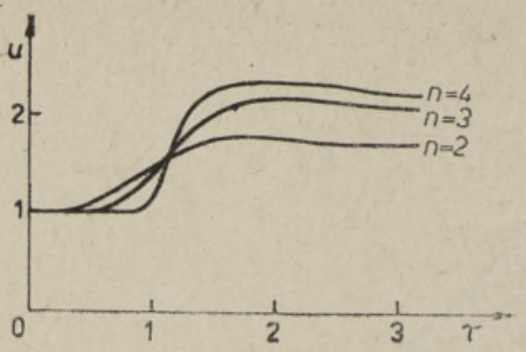

Рис, 5. Изменение максимальной амплитуды профиля волны.

Картина эволюции профилей волн при значениях $\mu=-0,15$ и $-0,0222$ (рис. 1 и 2) отчетливо характеризует образование солитонов, начиная с солитона с наибольшей амплитудой и кончая солитоном с наименьшей амплитудой. При этом скорости отдельных солитонов относительно базовой системы движущихся координат различаются. Профили волн в различные моменты времени (координаты) сопоставлены (рис. 3 и 4) для тех же значений параметра дисперсии. При этом $\tau \sim 1,0$ соответствует моменту, когда согласно нелинейной модели в недиспергирующей среде возникает ударная волна, и максимальное значение $\tau$ на рис. 3 и $4-$ моменту, когда образовалась полная совокупность солитонов при определенном значении $\mu$. Процесс образования солитонов можно условно разделить на три этапа. Вначале $(0<\tau<1)$ превалируют нелинейные эффекты и происходит укручение профиля. На втором этапе в зависимости от значения параметра $\mu$ подключаются дисперсионные эффекты и происходит существенный рост максимальной амплитуды волны. При бо́льших $\mu$ это происходит более плавно, чем при меньших. На третьем. этапе происходит окончательное формирование солитонов, при этом амплитуды уменьшаются и приближаются к определенным асимптотам. Этот процесс для солитонов с максимальной амплитудой и различным числом $n$ показан на рис. 5 для одного периода. При больших $\mu$ дисперсионные эффекты подключаются раньше, при малых - позже (при $\tau \geqslant 1)$. Это находится в полном согласии со структурой параметра $\mu$ по формуле (7).

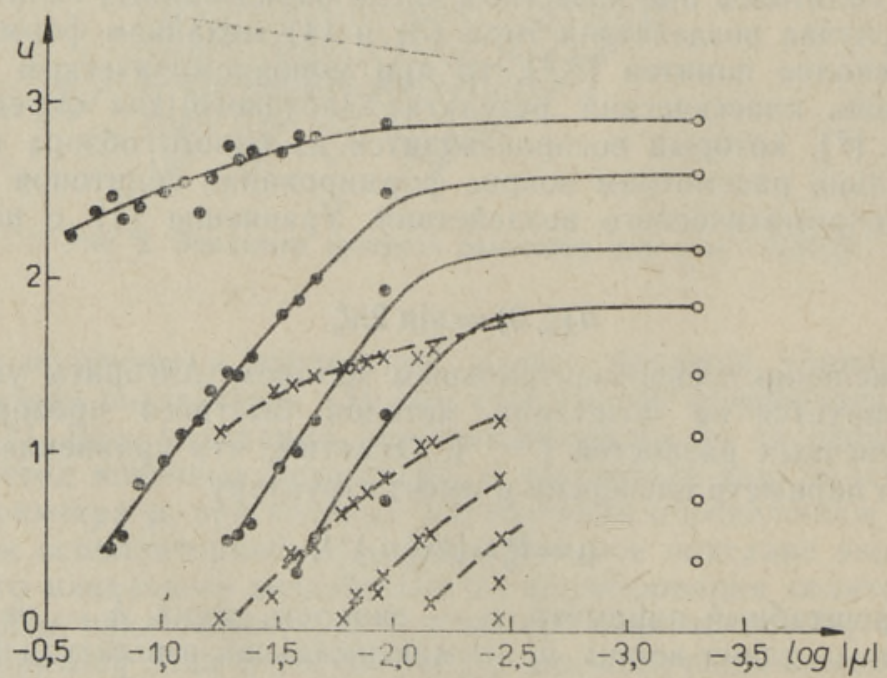

Рис. 6. Зависимость амплитуды солитонов от параметра дилперсин. - данные расчета для монохроматического воздействия (6), X - данные расчета для импульса (3)

$\left[{ }^{7}\right]$. О - данные расчета для монохроматического воздействия из [6]. 
А м плит уды солитонов. На рис. 6 представлены расчетные данные амплитуд для всех образующихся солитонов в зависимости от параметра дисперсии $\mu$. Для сравнения на этом же рисунке приведены результаты в случае воздействия (3), заимствованные из [ $\left.{ }^{7}\right]$. Как видно, общие закономерности при обеих воздействиях совпадают, несмотря на заметные существенные количественные различия. Наши расчеты и классические результаты из литературы [ $\left.{ }^{6}\right]$ : позволяют установить асимптотический характер роста амплитуд отдельных солитонов. При этом необходимо отметить значительный рост амплитуды наибольшего солитона по сравнению с амплитудой начального воздействия. Коэффициент усиления $u_{1} / u_{0}$ (где $u_{1}$ - максимальная амплитуда первого солитона, $u_{0}=1$ - амплитуда начального условия) в рассматриваемых случаях достигает 2,2-2,9. Кривые на рис. 6 (сплошные - воздействие (6) и прерывистые - воздействие (3)) являются в определенном смысле условными, из-за неполного соответствия моментов времени в расчетах. С такой же условностью получены и предыдущие результаты $\left[{ }^{7}\right]$. Значения параметра дисперсии $\mu$ (а также параметра $\mu^{-1 / 2}$, использованного в $\left.\left[{ }^{7}\right]\right)$, приводящие к образованию различного числа солитонов, приведены в таблице.

\begin{tabular}{|c|c|c|c|c|c|c|c|}
\hline \multirow{2}{*}{$\begin{array}{l}\text { Воз- } \\
\text { дей- } \\
\text { ствие }\end{array}$} & \multirow{2}{*}{$\begin{array}{l}\text { Пара- } \\
\text { метр }\end{array}$} & \multicolumn{6}{|c|}{ Число солитонов } \\
\hline & & 2 & 3 & 4 & 5 & 6 & 8 \\
\hline $\begin{array}{l}(6) \\
(3)\end{array}$ & $\begin{array}{l}|\mu| \\
\mu^{-1 / 2} \\
|\mu| \\
\mu^{-1 / 2}\end{array}$ & $\begin{array}{c}0,150-0,0417 \\
2,68-4,9 \\
0,0625-0,0204 \\
4-7\end{array}$ & $\begin{array}{c}0,0417-0,0222 \\
4,9-6,7 \\
0,024-0,0826 \\
7-11\end{array}$ & $\mid \begin{array}{c}0,0222-0,0111 \\
6,7-9,5 \\
0,0826 \\
11\end{array}$ & $\begin{array}{l}0,0111 \\
9,5\end{array}$ & $\begin{array}{l}0,0391 \\
16\end{array}$ & $\begin{array}{l}0,000493 \\
45\end{array}$ \\
\hline
\end{tabular}

Число солитонов существенно зависит не только от значения параметра $\mu$, но и от начального воздействия.

Нулевая линия. При воздействиях (3) и (4) численные расчеты дали значение амплитуды в бесконечности $u_{\infty}=0$. В случае же монохроматического воздействия (6) $u_{\infty} \neq 0$, и солитоны образуются на определенной нулевой линии (см. рис. 3,4$)$. В рассматриваемом интервале амплитуда $u_{\infty}$ остается постоянной и равняется приблизительно $-0,7$. Этот результат говорит о том, что в случае монохроматического начального воздействия фазы сжатия и разрежения искажаются по-разному (см. рис. 3 и 4 ). В случае $\mu<0$ фаза сжатия имеет бо́льшую абсолютную амплитуду, нежели фаза разрежения. Такая неравномерность искажения импульса имеет существенное значение при решении многих задач (задачи концентрации напряжений, нелинейной акустики и др.).

\section{ЛИТЕРА Т У РА}

1. Bishop, A. R., Krumhansl, J. A., Trullinger, S. E. Physica D, Nonlin. Phen., 1, № $1,1-44$ (1980).

2. Jefirey, A., Kakutani, T. SIAM Rev., 14, № 4, 582-643 (1979).

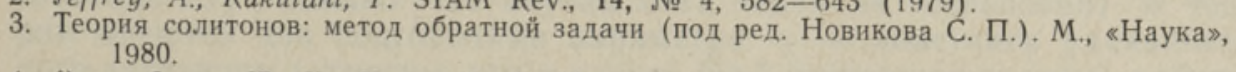

4. Энгельбрехт Ю. К., Нигул У. К. Нелинейные волны деформации. М., «Наука», 1981.

5. Korteweg, D. J., de Vries, G. Phil. Mag., 39, 422-443 (1895).

6. Zabusky, N. J. In: Proc. Symp. Nonlinear Partial Differential Equations (ed. by

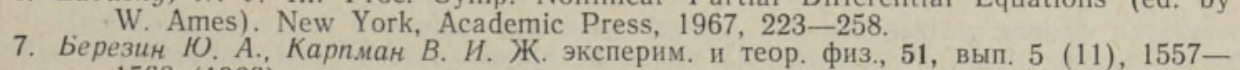
1568 (1966).

8. Березин Ю. А. Ж. техн. физ., 38, № 1, 24-27 (1968). 
9. Гасенко В. Г. В кн.: Исследования по гидродинамике и теплообмену. Новосибирек, «Наука», $1976,81-87$.

10. Fornberg, B., Whitham, G. B. Phil. Trans. Roy. Soc. London (A), 289, 373-404 (1978).

11. Пейпман Т. А., Энгельбрехт Ю. К. Уч. зап. Тартуск. ун-та, 627, 107-112 (1982).

Пнститут кибернетики

Академии наук Эстонской ССР

Поступила в редакцию $5 /$ IX 1983

\section{T. PEIPMAN, J. ENGELBRECHT}

\section{SOLITONIDE FORMEERUMISEST}

On uuritud solitonide formeerumist Korteweg-de Vriesi võrrandi baasil monokromaatilise algtingimuse korral ning vōrreldud tulemusi impulss-tüüpi algtingimusega lahendusega $\left[{ }^{7}\right]$ ja tuntud klassikalise lahendusega $\left[{ }^{6}\right]$. On kasutatud numbrilist algoritmi, mis põhineb kiirel Fourier' teisendusel ruumis ning lõplike vahede meetodil ajas [ ${ }^{11}$. On esitatud solitonide arvu ja amplituudide sõltuvus dispersiooni parameetrist $\mu$ ning detailselt näidatud solitonide formeerumise kolm etappi: 1) mittelineaarsete efektide etapp; 2) amplituudide järsu kasvu etapp, kus domineerib dispersioen; 3) solitonide lōpliku kujunemise etapp. Monokromaatilise algtingimuse puhul on protsessi iseärasu. seks nulljoone nihutus.

\section{T. PEIPMAN, J. ENGELBRECHT}

\section{ON FORMATION OF SOLITONS}

The formation of solitons modelled by the Korteweg-de Vries equation from certain initial conditions has been thoroughly investigated in case of pulse-like perturbations (3), (4) $[7,8]$. In this paper the formation of solitons in case of a monochromatic perturbation (6) is investigated in detail and matched with the classical result of Zabusky $\left[{ }^{6}\right]$. The results are obtained using the numerical algorithm based on the pseudospectral (Fast Fourier Transiorm) treatment of the space dependence together with a leap-frog scheme in time $\left[{ }^{11}\right]$. The number of the solitons and their amplitudes depend on the dispersion parameter $\mu$ (Fig. 1-4). The results of the numerical experiment are given in the Table together with the results obtained for the pulse-like perturbation (3) $\left[{ }^{7}\right]$. The formation process is characterized by three stages: 1) the stage of pulse distortion (nonlinear effects); 2) the stage of fast increase in amplitude (dispersion effects); 3) the final formation of solitons (see Fig. 5). The amplitudes during the second stage are, generally speaking, bigger than the amplitude of a formed soliton. The amplitudes of the whole sequences of solitons depending on the dispersion parameter $\mu$ are shown in Fig. 6. The amplitude of the zero-line $u_{\infty}$ (the amplitude at the infinity) is almost constant for the investigated values of dispersion parameter $\mu$. In several applications as, for example, nondestructive testing, acoustic resonators and propagation in seismic wavelets, the process of the formation of solitons deserves the full attention. 